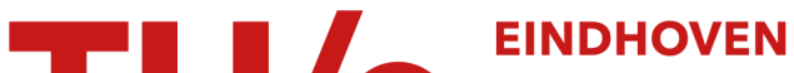

\section{A numerical test of long-time stability for rigid body integrators}

Citation for published version (APA):

Ortolan, G., \& Saccon, A. (2012). A numerical test of long-time stability for rigid body integrators. International Journal for Numerical Methods in Engineering, 90(3), 390-402. https://doi.org/10.1002/nme.3333

DOI:

10.1002/nme.3333

Document status and date:

Published: 01/01/2012

\section{Document Version:}

Publisher's PDF, also known as Version of Record (includes final page, issue and volume numbers)

\section{Please check the document version of this publication:}

- A submitted manuscript is the version of the article upon submission and before peer-review. There can be important differences between the submitted version and the official published version of record. People interested in the research are advised to contact the author for the final version of the publication, or visit the $\mathrm{DOI}$ to the publisher's website.

- The final author version and the galley proof are versions of the publication after peer review.

- The final published version features the final layout of the paper including the volume, issue and page numbers.

Link to publication

\section{General rights}

Copyright and moral rights for the publications made accessible in the public portal are retained by the authors and/or other copyright owners and it is a condition of accessing publications that users recognise and abide by the legal requirements associated with these rights.

- Users may download and print one copy of any publication from the public portal for the purpose of private study or research.

- You may not further distribute the material or use it for any profit-making activity or commercial gain

- You may freely distribute the URL identifying the publication in the public portal.

If the publication is distributed under the terms of Article 25fa of the Dutch Copyright Act, indicated by the "Taverne" license above, please follow below link for the End User Agreement:

www.tue.nl/taverne

Take down policy

If you believe that this document breaches copyright please contact us at:

openaccess@tue.nl

providing details and we will investigate your claim. 


\title{
A numerical test of long-time stability for rigid body integrators
}

\author{
Giulia Ortolan ${ }^{1, *, \dagger}$ and Alessandro Saccon ${ }^{2, \dagger}$ \\ ${ }^{1}$ Department of Information Engineering, University of Padova, via Gradenigo, 6/B, 35131 Padova, Italy \\ ${ }^{2}$ Instituto Superior Técnico, Technical University of Lisbon, Av. Rovisco Pais, 1, 1049-001 Lisboa, Portugal
}

\begin{abstract}
SUMMARY
In the context of Hamiltonian ODEs, a necessary condition for an integrator to be symplectic or conjugatesymplectic is that it nearly preserves the exact Hamiltonian. This paper introduces a numerical test of this necessity for rigid body methods. It turns out that several rigid body integrators proposed in literature fail this test. Hence, these integrators should be used with caution for long-time simulation. Copyright (C) 2011 John Wiley \& Sons, Ltd.
\end{abstract}

Received 10 December 2010; Revised 29 August 2011; Accepted 14 September 2011

KEY WORDS: electromechanical; variational methods; rigid body dynamics; geometric methods; long-time stability

\section{INTRODUCTION}

Rigid body dynamics plays a central role in many engineering branches such as civil, mechanics, and control engineering, as well as other important scientific disciplines such as physics and chemistry. Obtaining fast and accurate numerical integration schemes for long-time simulation of rigid-body type mechanical systems (e.g., in celestial mechanics and molecular dynamics) is an active area of research. One of the challenging aspects in designing an integration scheme for rigid body dynamics is that the differential equations are defined on a curved space, a Lie group, which is a smooth manifold possessing a group structure.

A geometric integrator is a numerical integrator that guarantees that each iterate remains on the smooth manifold on which the dynamics is intrinsically defined. A large number of geometric integrators for time integration of rigid body rotational dynamics have been proposed in the last 30 years. Some of these algorithms [1-6] are symplectic [7] in the sense that they preserve exactly the canonical symplectic form by construction, and therefore their good long-time behavior is assured by backward error analysis [8]. Some other algorithms [9-15] have been obtained by approximating the continuous time dynamics using ad hoc methods with the declared aim of obtaining computationally fast algorithms with small error constants. These latter algorithms seem to preserve the energy almost exactly over long periods of time, as well as a symplectic integrator would do. But the long-time behavior of these algorithms has been assessed using a set of numerical experiments, rather than theoretical justifications. Therefore, there is no guarantee of long-time energy stability in situations that differ from those tested.

Being derived using ad hoc methods, it is not clear which of these geometric integrators are in fact symplectic. In addition, they could nearly preserve the energy over a long time being conjugatesymplectic [16] like Newmark algorithm on vector spaces (see [17] and references therein). This

\footnotetext{
*Correspondence to: Giulia Ortolan, Department of Information Engineering, University of Padova, via Gradenigo, 6/B, 35131 Padova, Italy.

†E-mail: g.ortolan@libero.it

\#E-mail: asaccon@isr.ist.utl.pt 
motivated us to design a simple test (in the spirit of [18]) that allows one to conclude that a geometric rigid body integrator is neither symplectic nor conjugate-symplectic, whenever the test failed. This conclusion is drawn using backward error analysis of a symplectic integrator on a simply connected region of the phase space [8]. A main result of this paper is that many rigid body integrators proposed in the literature $[9,13-15]$ fail this test, showing that these algorithms can inject or dissipate energy artificially.

The test consists in integrating the rotation dynamics of a rigid body in a suitable static potential field. Since the methods we have tested are symmetric, and the test defines a reversible Hamiltonian system, this is also an interesting counterexample in the context of Lie groups to the belief that symmetric methods applied to reversible Hamiltonian systems nearly preserve the energy over long times. A preliminary version of this work has been presented in [19].

This paper is organized as follows. A literature review on conservative rigid body integrators is provided in Section 2. The basics of rigid body dynamics and the notation that we will use throughout the paper is discussed in Section 3. In Section 4, we describe the stress test that we apply to a selection of (recent and classical) integration schemes that have been proposed for long-time simulation of rigid body rotational dynamics. The rigid body methods we have tested will be described in Section 5. Numerical results of the test are discussed in Section 6. Finally, conclusions are drawn in Section 7.

\section{A REVIEW OF INTEGRATORS FOR CONSERVATIVE RIGID BODY SYSTEMS}

In this Section, we present a survey of the literature about integrators for conservative rigid body systems. This survey is limited to research papers that address the problem of integrating the dynamics of a rigid body in a generic configuration-dependent potential field.

Since the flow of a Hamiltonian system (such as that of a rigid body in a potential field) preserves the associated Hamiltonian, the canonical symplectic form, and (if a group symmetry is present) the corresponding momentum map, we will emphasize the behavior of the methods related to the preservation of these quantities. We group the algorithms into two categories: those approximating the flow of the equations of motion and those approximating the variational principle from which the Hamiltonian differential equations arise. The algorithms that belong to the second class are symplectic by construction; the first class of algorithms is instead more varied, with some algorithms known to be symplectic, others known to be not, and some others claimed to be based on numerical simulations. This latter group is the main object of investigation of this paper.

\section{Approximation of Hamiltonian differential equations.}

Simo and collaborators have developed a substantial body of work on rigid body integrators $[1,9,10]$. This work was originally motivated by the need to develop conserving algorithms that efficiently simulate the structural dynamics of rods and shells. In the first paper [9, Section 3], the classical Newmark scheme for integration of mechanical systems is extended to the rigid body configuration space, the Lie group $\mathrm{SO}(3)$. It was not apparent to these investigators if the proposed Lie-Newmark method has the necessary structure-preserving properties. Later, Simo and Wong [10, Section 6] prove that the Lie-Newmark method neither exactly preserve energy nor conserve momentum for the free rigid body; in the same paper, they propose other algorithms (Algo_1 [10, Section 4] and Algo_C1 [10, Section 5]), proving that, in the free rigid body case, both preserve energy whereas only the latter preserves momentum enforcing its rate balance at an intermediate time step; none of these algorithms is nevertheless symplectic ([10, Section 1] and [11, Section 1]).

Afterwards, Austin et al. [11] understand that the midpoint rule applied to Euler's equations with a Cayley reconstruction procedure is, in fact, a simple non-symplectic energy-momentum-preserving method for $\mathrm{SO}$ (3) (the method is not a member of the Lie-Newmark family introduced in [9]). In [1, Section 3], Lewis and Simo present a symplectic, energy and momentum-preserving integrator for the free rigid body, which encompasses, as a particular case, the energy-momentum algorithms of Simo and Wong [10] and the midpoint-rule integrator of Austin et al. [11], with the further property 
that the scheme is symplectic. Unfortunately, the algorithm of Lewis and Simo loses its preservation properties for a generic potential, although it might be symplectic or energy preserving for some particular choice of the potential energy and in presence of specific group symmetries (e.g., in the case of a heavy top [1, Section 4]).

In [13, Section 3], Krysl introduces an integration algorithm (LIEMID[EA]), which is explicit in the torque evaluation and momentum-preserving (only for the free rigid body). The paper proposes a series of numerical tests (namely, free rigid body, fast and slow Lagrangian tops, and rigid body in Coulomb potential with soft wall contact) showing that the accuracy of LIEMID[EA] method outperforms the algorithms by Simo and Wong [10], Austin et al. [11], and Krysl and Endres [12]. Based on numerical evidence of bounded energy error, the algorithm is claimed to be symplectic. In a later work [20], Krysl proposes a momentum-preserving form of the trapezoidal rule (TRAPM) and of the midpoint rule (IMIDM) for the rigid body dynamics. These methods are mutually conjugate, and in several numerical tests they exhibit a bounded energy error and a higher accuracy than the algorithms by Simo and Wong [10] and Austin et al. [11].

In [14], Nukala and Shelton propose two schemes (PRK and MCG), both of which can be thought as splitting methods, based on the ideas of partitioned Runge-Kutta and Crouch-Grossman methods. In the free rigid body case, the algorithms exactly preserve the momentum, and PRK is almost Poisson [14, Section 3]. According to the authors [14, Section 4], the numerical results show that those methods exhibit superior performance compared with the algorithms of Simo and Wong [10], Lie-Newmark [9], Moser and Veselov [2], Lewis and Simo [1], and Krysl [13]. The tests are conducted on the free rigid body, heavy top, and fast and slow Lagrangian tops.

Recently, Koziara and Bićanić [15] proposed a computationally simple explicit method for the rigid body suited for short-term simulations and constrained mechanical systems. For long-time simulations, the authors propose a semi-explicit version (NEW3), which introduces a slight increase in the computational complexity [15, Section 3], while preserving - in the free rigid body case the momentum.

\section{Approximation of Hamiltonian variational principle.}

In a famous paper, Moser and Veselov [2] derive an integrator for the free rigid body by embedding $\mathrm{SO}(3)$ in the linear space of $3 \times 3$ matrices and using Lagrange multipliers to constrain the body configuration to $\mathrm{SO}(3)$. The discrete Moser-Veselov is a particular case of the RATTLE algorithm for matrix Lie groups [4,21]. The RATTLE scheme is a classical method for the integration of constrained Hamiltonian systems. Its application to matrix Lie group and, in particular to rigid body integration, was proposed independently by Reich [3] and McLachlan and Scovel [4]. This method is symplectic and momentum-preserving both for the free body case and the generic potential case, and exactly preserves energy only in the former case [1, Section 1].

Based on the new approach to symplectic integration proposed by Veselov [2, 22], who developed a discrete mechanics using a discretization of Hamilton's principle, Marsden and collaborators $[23,24]$ have formalized the concept of discrete Lagrangian and discrete Euler-Lagrange equations. This method leads in a natural way to symplectic and momentum-preserving (symplecticmomentum) integrators. In [25], an approach for deriving symplectic rigid body integrators is presented in the context of orbital mechanics. In [5], a Runge-Kutta type discretization of the Hamilton-Pontryagin principle for a mechanical system on Lie groups is proposed. This yields, when applied to a rigid body system, to a class of second-order integrators that resemble the Lie-Newmark method but are symplectic-momentum for a generic potential.

For completeness of exposition, we want to emphasize that variational integration theory is not the only available framework to systematically generate symplectic integrators. Another well known and powerful idea is that of splitting (see, e.g., [26]). We refer the reader to [27] and references therein for an up-to-date discussion on the use of splitting methods for the integration of rigid body dynamics. A discussion on the connection between splitting methods and variational integrators goes beyond the scope of this work.

For the reader's convenience, we organize in Table I a list of integrators for conservative rigid body systems with a generic potential. In the first three columns, we report the name of the method 
Table I. Synoptic table of the rigid body integrators and their preserving properties.

\begin{tabular}{|c|c|c|c|c|c|c|c|c|}
\hline \multirow[t]{2}{*}{ Algorithm } & \multirow[t]{2}{*}{ Ref. } & \multirow[t]{2}{*}{ Year } & \multicolumn{3}{|c|}{ Free rigid body } & \multicolumn{3}{|c|}{ Rigid body with generic potential } \\
\hline & & & Symplectic & Energy & Momentum & Symplectic & Energy & Momentum \\
\hline Lie-Newmark & [9] & 1988 & ? & & & ? & & \\
\hline Algo_1 & [10] & 1991 & & $\checkmark$ & & & ? & \\
\hline Algo_C1 & [10] & 1991 & & $\checkmark$ & $\checkmark$ & & ? & ? \\
\hline Austin et al. & [11] & 1993 & & $\checkmark$ & $\checkmark$ & & ? & ? \\
\hline Lewis \& Simo & [1] & 1994 & $\checkmark$ & $\checkmark$ & $\checkmark$ & & & \\
\hline RATTLE & {$[3,4]$} & 1994 & $\checkmark$ & $\checkmark$ & $\checkmark$ & $\checkmark$ & nearly & $\checkmark$ \\
\hline Variational & [23] & 1998 & $\checkmark$ & nearly & $\checkmark$ & $\checkmark$ & nearly & $\checkmark$ \\
\hline LIEMID(EA) & [13] & 2005 & ? & & $\checkmark$ & ? & & \\
\hline PRK & [14] & 2007 & & $\checkmark$ & $\checkmark$ & & & ? \\
\hline MCG & [14] & 2007 & ? & & $\checkmark$ & ? & & \\
\hline NEW3 & [15] & 2010 & ? & & $\checkmark$ & ? & & \\
\hline
\end{tabular}

as it appeared on the original paper (if no name was given, just the authors' name), corresponding reference, and year of publication. We highlight if the method preserves the canonical symplectic form, energy, and spatial momentum, both for the case of a free rigid body and for the case of a rigid body in a generic potential. For this latter case, an integrator marked as momentum-preserving conserves the momentum maps arising from a Lie group symmetry. Only for the energy, some of the entries are marked "nearly", meaning that the error on the preserved quantity is bounded over exponentially long times. A question mark denotes that the conservation of that quantity is not stated nor easily inferred for the corresponding integrator. Note that, in a few cases, the methods are symplectic, momentum preserving, and energy preserving: this implies that they are integrating exactly the Hamiltonian system up to a reparametrization of time [28].

\section{MATHEMATICAL PRELIMINARIES}

In this Section, we recall the rotational dynamics of a rigid body in a static potential field. The attitude of a rigid body can be parameterized using a $3 \times 3$ rotation matrix. The set of rotation matrices, together with the binary operation given by the standard matrix multiplication, forms the Lie group $\mathrm{SO}(3)$. Denote by $\mathbf{Q}(t) \in \operatorname{SO}(3), \boldsymbol{\Omega}(t)=\left[\Omega_{1}(t) \Omega_{2}(t) \Omega_{3}(t)\right]^{T} \in \mathbb{R}^{3}$, and $\mathbf{J}=\operatorname{diag}\left(J_{1}, J_{2}, J_{3}\right) \in \mathbb{R}^{3 \times 3}$ the configuration, body angular velocity and (time-independent) bodyfixed inertia tensor, respectively. Let $\tau: \mathrm{SO}(3) \rightarrow \mathbb{R}^{3}$ be a configuration-dependent torque acting on the body expressed in body coordinates and let $\widehat{\imath}: \mathbb{R}^{3} \rightarrow \mathbb{R}^{3 \times 3}$ be the map defined as

$$
\widehat{\boldsymbol{\Omega}}=\left[\begin{array}{ccc}
0 & -\Omega_{3} & \Omega_{2} \\
\Omega_{3} & 0 & -\Omega_{1} \\
-\Omega_{2} & \Omega_{1} & 0
\end{array}\right] .
$$

In terms of this notation, the governing equations are

$$
\left\{\begin{array}{l}
\dot{\mathbf{Q}}=\mathbf{Q} \widehat{\mathbf{\Omega}}, \quad \text { (reconstruction equation) } \\
\mathbf{J} \dot{\mathbf{\Omega}}=\mathbf{J} \mathbf{\Omega} \times \mathbf{\Omega}+\boldsymbol{\tau}(\mathbf{Q}), \quad \text { (Euler's equation) }
\end{array}\right.
$$

with initial conditions $(\mathbf{Q}(0), \boldsymbol{\Omega}(0))=\left(\mathbf{Q}_{0}, \boldsymbol{\Omega}_{0}\right) \in \mathrm{SO}(3) \times \mathbb{R}^{3}$.

We assume that the rotation dynamics derives from a (left-trivialized) Lagrangian $L: \operatorname{SO}(3) \times$ $\mathbb{R}^{3} \rightarrow \mathbb{R}$ of the form

$$
L(\mathbf{Q}, \boldsymbol{\Omega})=T(\boldsymbol{\Omega})-U(\mathbf{Q}),
$$


where $T(\boldsymbol{\Omega})=\frac{1}{2} \boldsymbol{\Omega}^{T} \mathbf{J} \boldsymbol{\Omega}$ is the kinetic energy, and $U(\mathbf{Q})$ is the potential energy. For this to hold, the torque $\boldsymbol{\tau}(\mathbf{Q})$ that appears in Equation (1b) has to be obtained from the directional derivative of the potential energy $U$ at $\mathbf{Q}$ in the direction $\mathbf{Q} \widehat{y}$, that is

$$
\boldsymbol{\tau}(\mathbf{Q})^{T} y=-\mathrm{D} U(\mathbf{Q}) \cdot \mathbf{Q} \hat{y}, \quad y \in \mathbb{R}^{3} .
$$

We recall that through the (left-trivialized) Legendre transform

$$
\Pi=\frac{\partial}{\partial \boldsymbol{\Omega}} L(\mathbf{Q}, \boldsymbol{\Omega})=\mathbf{J} \boldsymbol{\Omega},
$$

one derives the (left-trivialized) Hamiltonian

$$
H(\mathbf{Q}, \boldsymbol{\Pi})=\frac{1}{2} \boldsymbol{\Pi}^{T} \mathbf{J}^{-1} \boldsymbol{\Pi}+U(\mathbf{Q})
$$

expressed in term of the configuration $\mathbf{Q}$ and body angular momentum $\Pi$. From the Hamiltonian, one can then derive a set of Hamiltonian equations equivalent to Equation (1) (see, e.g., [7]).

Note that $H$ is separable and defines a reversible Hamiltonian system [16], because $H(\mathbf{Q},-\boldsymbol{\Pi})=H(\mathbf{Q}, \boldsymbol{\Pi})$. As in all Hamiltonian systems, the exact continuous-time flow of Equation (1) is symplectic and preserves the Hamiltonian $H$ [7]. Through the Legendre transform, this also implies that the energy $E(\mathbf{Q}, \boldsymbol{\Omega})=T(\boldsymbol{\Omega})+U(\mathbf{Q})$ is preserved.

\section{Remark}

Note that in Equation (1), we are using the realization of the cotangent bundle $T^{*} \mathrm{SO}(3)$ as the product $\mathrm{SO}(3) \times \mathbb{R}^{3}$, obtained via left translation and successive identification of the dual of Lie algebra $\mathfrak{S D}(3)^{*}$ with $\mathbb{R}^{3}$. More details on this well-known fact and, in particular, the relationship between the canonical symplectic form on $T^{*} \mathrm{SO}(3)$ and the equivalent (non-canonical) symplectic form on $\mathrm{SO}(3) \times \mathbb{R}^{3}$ can be found, for example, in [29, Chapter 12].

\section{DESCRIPTION OF THE NUMERICAL TEST}

The numerical experiment that we introduced is a necessary test for the underlying symplecticity of a Lie group method, in the sense that, if an integrator exhibits a systematic drift in total energy, one can conclude that it is neither a symplectic nor a conjugate-symplectic integrator for Equation (1). As mentioned in Section 1, this experiment has proven to be able to detect an energy drift in a series of algorithms that, on the contrary, show an excellent long-time behavior in standard tests.

The test we propose in this Section is strongly inspired by a numerical experiment reported in $[18, \S 4.4]$ even though, unfortunately, it does not possess a similar simple and clear physical interpretation. In [18], a systematic energy drift of a fourth-order accurate, implicit, and symmetric Lobatto IIIB scheme is shown for the integration of the dynamics of a spring pendulum with exterior forces.

Define the function dist : $\mathrm{SO}(3) \times \mathrm{SO}(3) \rightarrow \mathbb{R}$ as

$$
\operatorname{dist}\left(\mathbf{Q}_{1}, \mathbf{Q}_{2}\right):=\sqrt{2 \operatorname{tr}\left(I-\mathbf{Q}_{1}^{T} \mathbf{Q}_{2}\right)},
$$

with $I \in \mathrm{SO}(3)$ the identity matrix. Recalling that the Frobenius matrix norm is defined as $\|A\|_{F}:=$ $\sqrt{\operatorname{tr}\left(A^{T} A\right)}$, for $A \in \mathbb{R}^{n \times n}$, it is straightforward to verify that $\operatorname{dist}(\cdot, \cdot)$ defines the metric on $\operatorname{SO}(3)$ induced by the Frobenius norm (use the identity $\left\|\mathbf{Q}_{2}-\mathbf{Q}_{1}\right\|_{F}^{2}=2 \operatorname{tr}\left(I-\mathbf{Q}_{1}^{T} \mathbf{Q}_{2}\right)$ ).

We consider a single rigid body in a static field defined by the potential energy function $U_{\alpha}: \mathrm{SO}(3) \rightarrow \mathbb{R}$ given by

$$
U_{\alpha}(\mathbf{Q})=(\operatorname{dist}(\mathbf{Q}, I)-1)^{2}-\frac{\alpha}{\operatorname{dist}\left(\mathbf{Q}, \mathbf{Q}_{m}\right)} .
$$


The first term in the right hand side of Equation (3) is a bounded potential, which attains its minimum value at all $\mathbf{Q} \in \mathrm{SO}(3)$ satisfying $\operatorname{dist}(\mathbf{Q}, I)=1$. The second term is an unbounded potential that generates an attraction toward the configuration $\mathbf{Q}_{m} \in \mathrm{SO}(3)$, whose exact value will be discussed in the following as well as that of the tuning parameter $\alpha$.

For $\alpha=0$, the potential energy $U_{\alpha=0}$ achieves its minimum value on the two-dimensional surface

$$
S:=\{\mathbf{Q} \in \operatorname{SO}(3): \operatorname{dist}(\mathbf{Q}, I)=1\} .
$$

This implies that the set $S \times\{\mathbf{0}\} \subset \mathrm{SO}(3) \times \mathbb{R}^{3}$ is a (locally) stable set in the sense of Lyapunov for the dynamics of the rigid body, as we know from classical mechanics. One can prove this fact using the energy, that is,

$$
E(\mathbf{Q}, \boldsymbol{\Omega})=T(\boldsymbol{\Omega})+U_{\alpha=0}(\mathbf{Q})
$$

as Lyapunov function and noting that, for every $\bar{E} \geqslant 0$, the set $N(\bar{E}):=\{(\mathbf{Q}, \boldsymbol{\Omega}) \in \operatorname{SO}(3) \times$ $\left.\mathbb{R}^{3} \mid E(\mathbf{Q}, \boldsymbol{\Omega}) \leqslant \bar{E}\right\}$ is a compact neighborhood of $S$ reducing to $S$ for $\bar{E} \rightarrow 0$. It is also important to note that the set $N(\bar{E})$, for $\bar{E}>0$ small, is simply connected. This follow from the fact that the set of minima of $U_{\alpha=0}(\mathbf{Q})$ is simply connected (it can be pictured as a sphere of radius one when employing the exponential coordinates as local coordinates for $\mathrm{SO}(3)$ ) and from the fact that the kinetic energy is a quadratic function.

For $\alpha>0$, the set $S$ gets perturbed by the unbounded attractive potential. On this perturbed energy landscape, the rigid body experiences an attraction toward the configuration $\mathbf{Q}_{m}$. Yet, if we place the attraction point $\mathbf{Q}_{m}$ sufficiently far from the set $S$ and choose the tuning parameter $\alpha>0$ sufficiently small, the set $S$ gets only slightly perturbed into a new set that we label $S_{\alpha}$. Furthermore, the set $S_{\alpha} \times\{\mathbf{0}\} \subset \mathrm{SO}(3) \times \mathbb{R}^{3}$ is locally Lyapunov stable like the unperturbed set $S \times\{\mathbf{0}\}$. The existence of an invariant simply connected set, like $N(\bar{E})$, containing $S_{\alpha} \times\{\mathbf{0}\}$ also follows.

In summary, we can design $U_{\alpha}$ so that the true solution is confined to a simply connected neighborhood of the set $S_{\alpha} \times\{\mathbf{0}\}$ of approximately known shape. The closest the initial conditions are to the set of stable equilibria $S_{\alpha} \times\{\mathbf{0}\}$, the smaller the deviation from it is (Lyapunov stability).

Recall that a symplectic integrator is interpolated by a level set of a modified energy function nearby the true energy $[8,16,30]$. However, the existence of a globally defined modified energy function might require some additional conditions unless the phase space is simply connected, as discussed in [8, Remark after Proposition 1] and also in [31]. Regarding this example, since the Lie group $\mathrm{SO}(3)$ is not simply connected, the existence of a globally defined modified energy cannot be assumed a priori. Nonetheless, the existence of a simply connected invariant neighborhood of $S_{\alpha} \times\{\boldsymbol{0}\}$ ensures the existence of a well-defined modified energy in that region. Therefore, the trajectory of a symplectic integrator with initial condition sufficiently close to $S_{\alpha} \times\{\mathbf{0}\}$ will remain in that neighborhood and will almost preserve the energy for exponentially long time.

\section{NUMERICAL INTEGRATORS}

We test seven different algorithms, most of them appeared in the literature in the last 10 years. Each subsection is devoted to the description of a different method. All the algorithms are detailed using the notation introduced in Section 3.

\subsection{Explicit Lie-Newmark method}

The Lie-Newmark family of integrators was proposed about 20 years ago in [9]. These methods consist of a Newmark-style discretization of Equation (1b) and a discretization of Equation (1a) that ensures that the configuration update remains on $\mathrm{SO}(3)$.

In this paper, we focus on two specific members of the Lie-Newmark family. The first one is the Lie group analogue of the so called explicit Newmark method on vector spaces (see [32, Chapter 9]), known as the Verlet integrator in molecular dynamics [16, Chapter I].

Given the timestep $h$ and the current configuration at the $k$-th instant of time $\left(\mathbf{Q}_{k}, \boldsymbol{\Omega}_{k}\right) \in$ $\mathrm{SO}(3) \times \mathbb{R}^{3}$, the explicit Lie-Newmark (ELN) scheme determines the update $\left(\mathbf{Q}_{k+1}, \boldsymbol{\Omega}_{k+1}\right)$ by the following iteration rule: 


$$
\left\{\begin{aligned}
\boldsymbol{\Omega}_{k+\frac{1}{2}} & =\boldsymbol{\Omega}_{k}+\frac{h}{2} \mathbf{J}^{-1}\left(\mathbf{J} \boldsymbol{\Omega}_{k} \times \boldsymbol{\Omega}_{k}+\boldsymbol{\tau}\left(\mathbf{Q}_{k}\right)\right) \\
\mathbf{Q}_{k+1} & =\mathbf{Q}_{k} \operatorname{cay}\left(h \boldsymbol{\Omega}_{k+\frac{1}{2}}\right) \\
\boldsymbol{\Omega}_{k+1} & =\boldsymbol{\Omega}_{k+\frac{1}{2}}+\frac{h}{2} \mathbf{J}^{-1}\left(\mathbf{J} \boldsymbol{\Omega}_{k+1} \times \boldsymbol{\Omega}_{k+1}+\tau\left(\mathbf{Q}_{k+1}\right)\right)
\end{aligned}\right.
$$

In Equation (4b), the Cayley map cay : $\mathbb{R}^{3} \rightarrow \mathrm{SO}(3)$ is defined as

$$
\operatorname{cay}(x)=I+\frac{4}{4+|x|} \hat{x}+\frac{2}{4+|x|} \hat{x}^{2}, \quad x \in \mathbb{R}^{3},
$$

where $I$ is the identity matrix. The Cayley map is a second-order approximation of the exponential map on $\mathrm{SO}(3)$. There are other maps that one can use in place of the Cayley map in Equation (4b) (see, e.g., [33, §5.4]), but the Cayley map is known to be computationally less expensive than the exponential map. As we will discuss in Section 6, we have noted no difference in the long-time behavior of the integrator when using the exponential map (as originally proposed in [9]) instead of the Cayley map.

The method is called explicit as it is explicit in the torque evaluation, although the integrator is in fact semi-explicit because the equation (4c) is implicit. The implicitness is not severe, however, as the method is only implicit in the angular velocity and not in the attitude.

\subsection{Trapezoidal Lie-Newmark method}

The second Lie-Newmark method that we consider in this paper is the so called Trapezoidal LieNewmark (TLN) algorithm, which is the Lie group analogue of the well-known trapezoidal rule on vector spaces. The second-order accuracy of this algorithm has been proved in [9]. Given $\left(\mathbf{Q}_{k}, \boldsymbol{\Omega}_{k}\right) \in \mathrm{SO}(3) \times \mathbb{R}^{3}$ and timestep $h$, the TLN iteration rule is given by

$$
\left\{\begin{array}{l}
\left(I+\frac{h}{2} \widehat{\boldsymbol{\Omega}}_{k+1}\right) \mathbf{J} \boldsymbol{\Omega}_{k+1}-\frac{h}{2} \boldsymbol{\tau}\left(\mathbf{Q}_{k+1}\right)=\left(I-\frac{h}{2} \widehat{\boldsymbol{\Omega}}_{k}\right) \mathbf{J} \boldsymbol{\Omega}_{k}+\frac{h}{2} \boldsymbol{\tau}\left(\mathbf{Q}_{k}\right), \\
\mathbf{Q}_{k+1}=\mathbf{Q}_{k} \operatorname{cay}\left(h \frac{\boldsymbol{\Omega}_{k}+\boldsymbol{\Omega}_{k+1}}{2}\right) .
\end{array}\right.
$$

This method is fully implicit, and it requires the computation of the first derivative of the torque expression when, for example, a Newton method is employed to solve the nonlinear set of equations. As for the ELN method, we have experienced no differences in the long-time behavior for the choice of the Cayley map instead of the exact exponential map in the reconstruction equation.

\subsection{Explicit Lie-midpoint algorithm}

In [13], Krysl derives the following Lie-midpoint (LIEMID[EA]) algorithm from the composition of a half-step of a first-order midpoint Lie method with its adjoint. This method is therefore symmetric and second-order accurate; besides this, in the free rigid body case, it preserves exactly the spatial angular momentum. Due to its good behavior in preserving the energy, in [13] it is claimed that the method is symplectic.

Given $\left(\mathbf{Q}_{k}, \boldsymbol{\Omega}_{k}\right) \in \operatorname{SO}(3) \times \mathbb{R}^{3}$ and timestep $h$, the algorithm determines $\left(\mathbf{Q}_{k+1}, \boldsymbol{\Omega}_{k+1}\right)$ using the following iteration rule: 


$$
\left\{\begin{array}{l}
\boldsymbol{\Theta}_{k+\frac{1}{2}}=\frac{h}{2} \mathbf{J}^{-1} \exp \left(-\frac{1}{2} \boldsymbol{\Theta}_{k+\frac{1}{2}}\right)\left(\mathbf{J} \boldsymbol{\Omega}_{k}+\frac{h}{2} \boldsymbol{\tau}\left(\mathbf{Q}_{k}\right)\right), \\
\mathbf{Q}_{k+\frac{1}{2}}=\mathbf{Q}_{k} \exp \left(\boldsymbol{\Theta}_{k+\frac{1}{2}}\right), \\
\boldsymbol{\Omega}_{k+\frac{1}{2}}=\mathbf{J}^{-1} \exp \left(-\boldsymbol{\Theta}_{k+\frac{1}{2}}\right)\left(\mathbf{J} \boldsymbol{\Omega}_{k}+\frac{h}{2} \boldsymbol{\tau}\left(\mathbf{Q}_{k}\right)\right), \\
\boldsymbol{\Theta}_{k+1}=\frac{h}{2} \mathbf{J}^{-1} \exp \left(-\frac{1}{2} \boldsymbol{\Theta}_{k+1}\right) \mathbf{J} \boldsymbol{\Omega}_{k+\frac{1}{2}}, \\
\mathbf{Q}_{k+1}=\mathbf{Q}_{k+\frac{1}{2}} \exp \left(\boldsymbol{\Theta}_{k+1}\right), \\
\boldsymbol{\Omega}_{k+1}=\mathbf{J}^{-1}\left(\exp \left(-\boldsymbol{\Theta}_{k+1}\right) \mathbf{J} \boldsymbol{\Omega}_{k+\frac{1}{2}}+\frac{h}{2} \boldsymbol{\tau}\left(\mathbf{Q}_{k+1}\right)\right),
\end{array}\right.
$$

where $\exp : \mathbb{R}^{3} \rightarrow \mathrm{SO}(3)$ is the matrix exponential map

$$
\exp (x)=I+\frac{\sin (|x|)}{|x|} \hat{x}+\frac{1-\cos (|x|)}{|x|^{2}} \hat{x}^{2}, x \in \mathbb{R}^{3} .
$$

The updates (7a) and (7d) are both implicit, and therefore the algorithm involves the solution of two nonlinear equations per step. Nevertheless, because they are not implicit in the body attitude, the implicitness is not hard. The four remaining updates are explicit.

\subsection{Partitioned Runge-Kutta Munthe-Kaas method}

In [14], Nukala and Shelton consider a partitioned Runge-Kutta Munthe-Kaas (PRK) method for the integration of rigid body dynamics. Runge-Kutta Munthe-Kaas methods are a generalization of Runge-Kutta methods for differential equations evolving on a Lie group. They were introduced by Munthe-Kaas in [34,35]. The algorithm presented in [14] is partitioned [16, Chapter 2] as the rigid body dynamics (1) is seen as the partitioned system of equations

$$
\left\{\begin{aligned}
\dot{\mathbf{Q}} & =f(\mathbf{Q}, \boldsymbol{\Omega}):=\mathbf{Q} \widehat{\boldsymbol{\Omega}}, \\
\mathbf{J} \dot{\boldsymbol{\Omega}} & =g(\mathbf{Q}, \boldsymbol{\Omega}):=\mathbf{J} \boldsymbol{\Omega} \times \mathbf{\Omega}+\boldsymbol{\tau}(\mathbf{Q}) .
\end{aligned}\right.
$$

The idea of a partitioned Runge-Kutta method consists of choosing two different Runge-Kutta methods and integrating the first variable (in our case, $\mathbf{Q}$ ) with the first method, and the second variable $(\boldsymbol{\Omega})$ with the second method. In [14], because of the nature of rigid body dynamics, two Runge-Kutta Munthe-Kaas methods are chosen, corresponding to a two-stage Lobatto IIIA and a two-stage Lobatto IIIB. The Butcher tableaus of these two methods are, respectively, given by

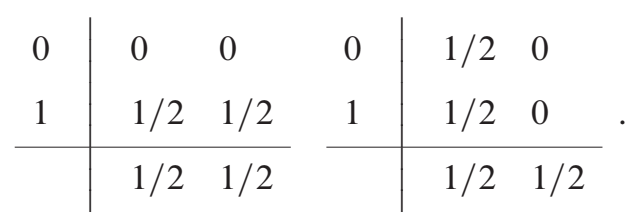

In the free rigid body case, this algorithm exactly preserves the momentum and is almost Poisson [14].

Using the notation introduced in Section 1, the partitioned Runge-Kutta Munthe-Kaas method is written as

$$
\left\{\begin{array}{l}
\boldsymbol{\Omega}_{k+\frac{1}{2}}=\boldsymbol{\Omega}_{k}+\frac{h}{2} \mathbf{J}^{-1}\left(\boldsymbol{\tau}\left(\mathbf{Q}_{k}\right)-\boldsymbol{\Omega}_{k+\frac{1}{2}} \times \mathbf{J} \boldsymbol{\Omega}_{k+\frac{1}{2}}\right) \\
\mathbf{Q}_{k+1}=\mathbf{Q}_{k} \operatorname{cay}\left(h \boldsymbol{\Omega}_{k+\frac{1}{2}}\right), \\
\boldsymbol{\Omega}_{k+1}=\boldsymbol{\Omega}_{k+\frac{1}{2}}+\frac{h}{2} \mathbf{J}^{-1}\left(\boldsymbol{\tau}\left(\mathbf{Q}_{k+1}\right)-\boldsymbol{\Omega}_{k+\frac{1}{2}} \times \mathbf{J} \boldsymbol{\Omega}_{k+\frac{1}{2}}\right)
\end{array}\right.
$$


This algorithm is explicit in the torque evaluation. The only implicitness is in computing the angular velocity at the midstep $k+1 / 2$ in Equation (9a). As a remark, we recall that the corresponding partitioned Runge-Kutta method on vector spaces is symplectic [36], although we also note that the proposed partitioned RKMK method does not follow the ideas presented in [37].

\subsection{Modified Crouch-Grossman method}

A splitting approach to the integration of Equation (1) is described by Nukala and Shelton in [14]. The rigid body dynamics is split into

$$
\left\{\begin{array}{l}
\dot{\mathbf{Q}}=\mathbf{Q} \widehat{\mathbf{\Omega}} \\
\mathbf{J} \dot{\mathbf{\Omega}}=0
\end{array}, \quad\left\{\begin{array}{l}
\dot{\mathbf{Q}}=0 \\
\mathbf{J} \dot{\boldsymbol{\Omega}}=(\mathbf{J} \boldsymbol{\Omega} \times \boldsymbol{\Omega})
\end{array} \quad, \quad\left\{\begin{array}{l}
\dot{\mathbf{Q}}=0 \\
\mathbf{J} \dot{\boldsymbol{\Omega}}=\boldsymbol{\tau}(\mathbf{Q})
\end{array}\right.\right.\right.
$$

The flow of the first and the third vector fields can be computed exactly, whereas for the second one, the authors propose an implicit scheme they refer to as implicit Crouch-Grossman (MCG) method [38,39]. The idea is simply to approximate the solution of the quasilinear system $\dot{Y}=A(Y) Y$ as $Y(h)=\exp (h A(Y(h))) Y(0)$ where, in our case, $Y=\mathbf{J} \boldsymbol{\Omega}$ and $A(Y)=-\hat{\boldsymbol{\Omega}}$. The three flow maps are then composed (according to splitting principles) with their adjoint methods in order to obtain a second-order symmetric reversible method. In [14], it is also proven that this method exactly preserves the spatial angular momentum for the rigid body.

Given $\left(\mathbf{Q}_{k}, \boldsymbol{\Omega}_{k}\right) \in \mathrm{SO}(3) \times \mathbb{R}^{3}$, the algorithm is given by

$$
\left\{\begin{array}{l}
\boldsymbol{\Omega}_{k+\frac{1}{2}}=\mathbf{J}^{-1}\left[\exp \left(-\frac{h}{2} \boldsymbol{\Omega}_{k+\frac{1}{2}}\right)\left(\mathbf{J} \boldsymbol{\Omega}_{k}+\frac{h}{2} \boldsymbol{\tau}\left(\mathbf{Q}_{k}\right)\right)\right] \\
\mathbf{Q}_{k+1}=\mathbf{Q}_{k} \exp \left(h \boldsymbol{\Omega}_{k+\frac{1}{2}}\right), \\
\boldsymbol{\Omega}_{k+1}=\mathbf{J}^{-1}\left[\exp \left(-h \boldsymbol{\Omega}_{k+\frac{1}{2}}\right)\left(\mathbf{J} \boldsymbol{\Omega}_{k}+\frac{h}{2} \boldsymbol{\tau}\left(\mathbf{Q}_{k}\right)\right)+\frac{h}{2} \boldsymbol{\tau}\left(\mathbf{Q}_{k+1}\right)\right] .
\end{array}\right.
$$

This integrator is semi-explicit because it is implicit only in the computation of angular velocity (10a).

\subsection{Koziara-Bićanić algorithm}

As introduced in Section 2, Koziara and Bićanić [15] propose a computationally simple explicit method suited for short-term simulations of constrained rigid-body type systems. The method is then modified obtaining a semi-explicit version (NEW3), which shows a significative improvement in the long-time behavior at the cost of a moderate increase in the computational complexity.

The algorithm is described by the following formulas:

$$
\left\{\begin{array}{l}
\mathbf{Q}_{k+\frac{1}{2}}=\mathbf{Q}_{k} \exp \left(\frac{h}{2} \boldsymbol{\Omega}_{k}\right), \\
\exp \left(\frac{h}{2} \boldsymbol{\Omega}_{k+1}\right) \mathbf{J} \boldsymbol{\Omega}_{k+1}=\exp \left(-\frac{h}{2} \boldsymbol{\Omega}_{k}\right) \mathbf{J} \boldsymbol{\Omega}_{k}+h \boldsymbol{\tau}\left(\mathbf{Q}_{k+\frac{1}{2}}\right), \\
\mathbf{Q}_{k+1}=\mathbf{Q}_{k+\frac{1}{2}} \exp \left(\frac{h}{2} \boldsymbol{\Omega}_{k+1}\right) .
\end{array}\right.
$$

Given $\left(\mathbf{Q}_{k}, \boldsymbol{\Omega}_{k}\right)$, in Equation (11a) the middle-step rotation $\mathbf{Q}_{k+1 / 2}$ is computed using a forward Lie-Euler method. Then, in Equation (11b), the angular velocity $\boldsymbol{\Omega}_{k+1}$ is computed using a midpoint approximation of the momentum equation (1b). Finally, in Equation (11c), a backward Lie-Euler method is used to compute $\mathbf{Q}_{k+1}$ from $\boldsymbol{\Omega}_{k+1}$. This algorithm is explicit in torque evaluation, and it is implicit only in the second step (11b), where the body angular velocity $\boldsymbol{\Omega}_{k+1}$ is computed. 
In the numerical tests reported in [15], the algorithm performs well, showing no energy drift. The authors therefore suggest that, as a result of its implementation simplicity, it might be interesting to study its stability properties.

\subsection{Variational Lie-Verlet method}

The variational Lie-Verlet (VLV) integrator was proposed in [5], and it is based on the theory of discrete and continuous Euler-Poincaré systems [23,40]. The method is closely related to, but different, from the RATTLE method for constrained mechanical systems [16]. Because of its variational nature, the method is symplectic by construction [5]. In the present context, this scheme is used to confirm that no energy drift can be observed using a symplectic integrator when integrating the rigid body dynamics with the static potential introduced in Section 4.

Given $\left(\mathbf{Q}_{k}, \boldsymbol{\Omega}_{k}\right) \in \operatorname{SO}(3) \times \mathbb{R}^{3}$ and timestep $h$, the Lie-Verlet algorithm determines $\left(\mathbf{Q}_{k+1}, \boldsymbol{\Omega}_{k+1}\right)$ by the following iteration rule:

$$
\left\{\begin{array}{l}
\boldsymbol{\Omega}_{k+\frac{1}{2}}=\boldsymbol{\Omega}_{k}+\frac{h}{2} \mathbf{J}^{-1}\left[\mathbf{J} \boldsymbol{\Omega}_{k+\frac{1}{2}} \times \boldsymbol{\Omega}_{k+\frac{1}{2}}-\frac{h}{2}\left(\boldsymbol{\Omega}_{k+\frac{1}{2}}^{T} \mathbf{J} \boldsymbol{\Omega}_{k+\frac{1}{2}}\right) \boldsymbol{\Omega}_{k+\frac{1}{2}}+\boldsymbol{\tau}\left(\mathbf{Q}_{k}\right)\right] \\
\mathbf{Q}_{k+1}=\mathbf{Q}_{k} \operatorname{cay}\left(h \boldsymbol{\Omega}_{k+\frac{1}{2}}\right) \\
\boldsymbol{\Omega}_{k+1}=\boldsymbol{\Omega}_{k+\frac{1}{2}}+\frac{h}{2} \mathbf{J}^{-1}\left[\mathbf{J} \boldsymbol{\Omega}_{k+\frac{1}{2}} \times \boldsymbol{\Omega}_{k+\frac{1}{2}}+\frac{h}{2}\left(\boldsymbol{\Omega}_{k+\frac{1}{2}}^{T} \mathbf{J} \boldsymbol{\Omega}_{k+\frac{1}{2}}\right) \boldsymbol{\Omega}_{k+\frac{1}{2}}+\boldsymbol{\tau}\left(\mathbf{Q}_{k+1}\right)\right]
\end{array}\right.
$$

This algorithm is semi-explicit: the updates (12b) and (12c) are explicit whereas (12a) is implicit only in the body velocity.

\section{NUMERICAL RESULTS}

In this Section, we present the numerical results obtained by using the methods detailed in Section 5 . The following set of parameters has been chosen after a series of preliminary tuning experiments. The inertia matrix is equal to

$$
\mathbf{J}=\left[\begin{array}{lll}
2 & 0 & 0 \\
0 & 2 & 0 \\
0 & 0 & 4
\end{array}\right]
$$

The initial attitude $\mathbf{Q}_{0} \in \mathrm{SO}(3)$ has been selected so that $\operatorname{dist}\left(\mathbf{Q}_{0}, I\right)$ is nearly one, whereas the initial velocity $\boldsymbol{\Omega}_{0} \in \mathbb{R}^{3}$ has been chosen relatively small. As described in Section 4 , this choice is motivated by the will of maintaining the system trajectories in a neighborhood of the set $S \times\{\mathbf{0}\} \subset \mathrm{SO}(3) \times \mathbb{R}^{3}$, when the tuning parameter $\alpha$ is zero. Specifically, the initial condition $\left(\mathbf{Q}_{0}, \boldsymbol{\Omega}_{0}\right)$ is

$$
\mathbf{Q}_{0}=\exp \left(v_{0}\right), v_{0}=\left[\begin{array}{c}
0 \\
0.7227 \\
0
\end{array}\right], \boldsymbol{\Omega}_{0}=\left[\begin{array}{c}
0 \\
0 \\
0.625
\end{array}\right]
$$

The tuning parameter $\alpha$ and the attraction point $\mathbf{Q}_{m}$ have been set to provide a weak perturbation of the original trajectory. The chosen values are $\alpha=0.3$ and

$$
\mathbf{Q}_{m}=\exp \left(v_{m}\right), v_{m}=\frac{1}{\sqrt{2}}\left[\begin{array}{c}
2.5 \\
0 \\
2.5
\end{array}\right]
$$


The simulation has been carried on a long-time interval [0,10000], with timesteps $h=0.125$ and $h=0.25$. All the methods have been coded embedding $\mathrm{SO}(3)$ into the vector space $\mathbb{R}^{9}$; in virtue of the discrete form of the reconstruction equation (1a) for all methods, the $\mathrm{SO}(3)$ constraint $\left\|\mathbf{Q}_{k}^{T} \mathbf{Q}_{k}-I\right\|=0$ is satisfied for all methods up to machine precision.

Results are shown in Figure 1. A systematic drift for all the methods but the VLV can be observed. Comparing the figures of the left side with those on the right side, we see that the drift is linear with respect to time. A finer analysis also shows that the drift is quadratic in the timestep $h$. We abbreviate this fact by saying that the total energy error behaves like $\mathcal{O}\left(T h^{2}\right)$. Positive (MCG, NEW3, LIEMID[EA]) and negative energy drifts (ELN, TLN, PRK) of different slope are observed. Further numerical tests, not reported, conducted using the exponential map in place of the Cayley map in the reconstruction equation of ELN and TLN also show a very similar drift in the energy. Finally, it is of interest to note that LIEMID[EA] exhibits the smallest slope constant (in absolute value).

The accuracy diagrams, shown in Figure 2, confirm that all the methods are second-order accurate. Figure 2(a) shows the global error in the attitude matrix at time $T=5$ for different timesteps. Figure 2(b) shows, similarly, the global error in body angular velocity. The reference solution was computed using the function ode 45 in MATLAB, with an absolute tolerance $10^{-14}$ and relative tolerance $2 \cdot 10^{-14}$. It is interesting that, despite its implicitness, TLN shows the same accuracy than that of ELN in this numerical test.

All the algorithms have been implemented in MATLAB using a standard Newton method to solve for the implicit steps. We observed that LIEMID[EA] and TLN, respectively, require about two and six (!) times the running time of the remaining algorithms.
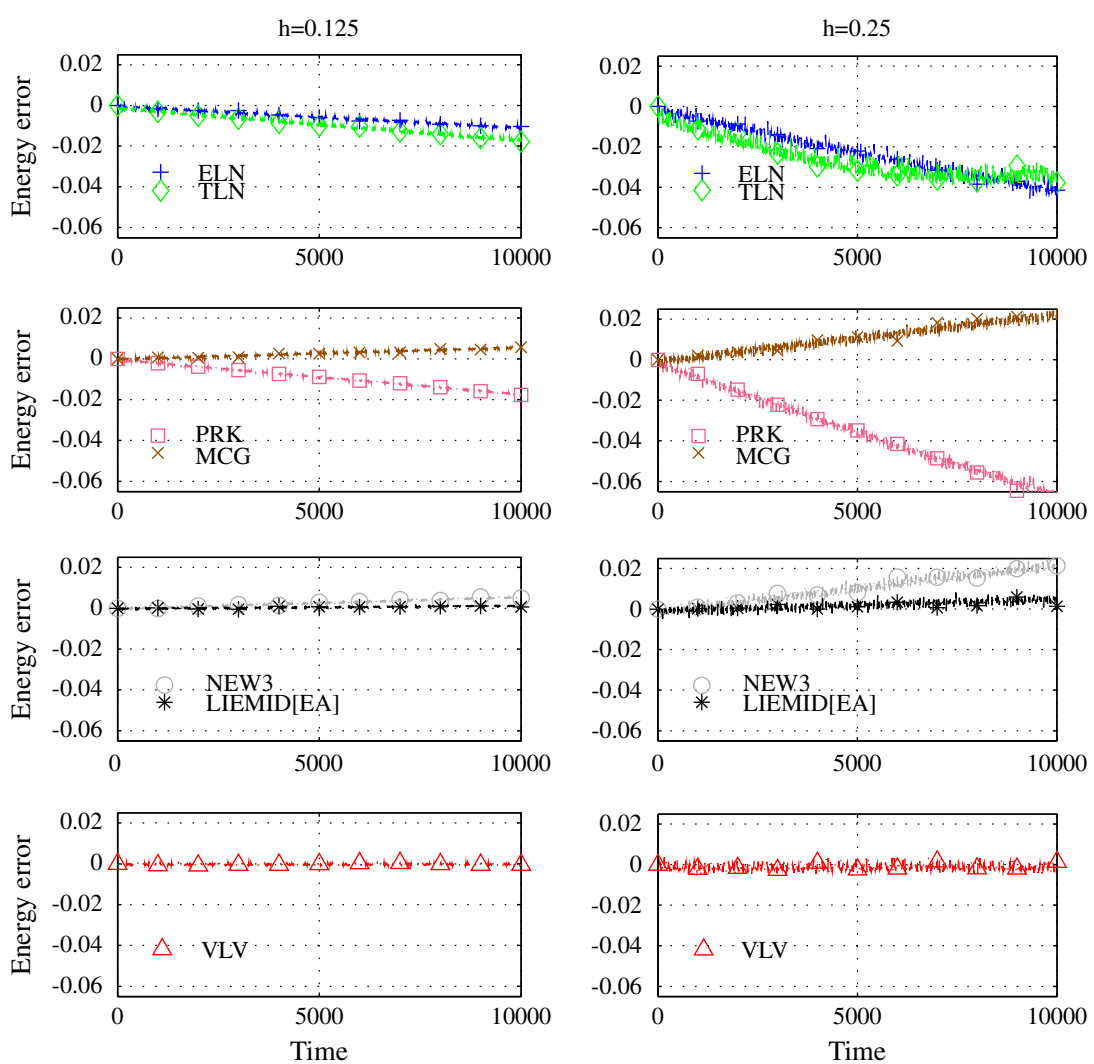

Figure 1. Energy error of the ELN, TLN, PRK, MCG, NEW3, LIEMID[EA], VLV algorithms. Plots on the left are obtained with a timestep $h=0.125$, plots on the right with a doubled timestep $h=0.25$. All the algorithms but VLV exhibit a systematic energy drift. On the other hand, the energy error of VLV method remains bounded as predicted by the theory. The initial conditions and parameters used are provided in the text. 

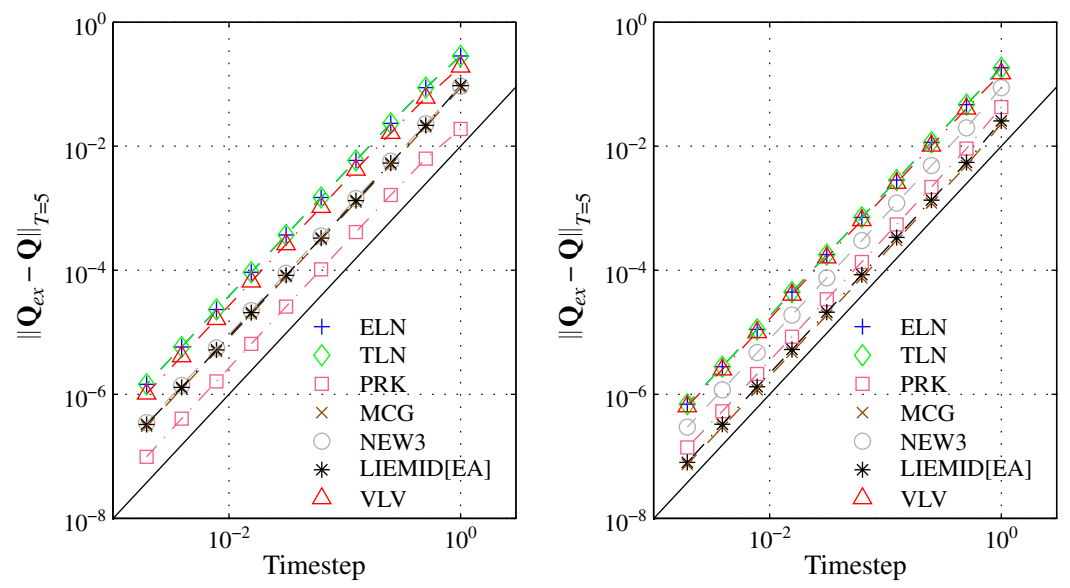

Figure 2. Global error of the ELN, TLN, PRK, MCG, NEW3, LIEMID[EA], VLV algorithms. The global error is evaluated in (a) configuration and (b) body angular velocity at a physical time of $T=5$ for timesteps $h \in\left\{1,2^{-1}, \ldots, 2^{-9}\right\}$. We use as a reference solution the integration of Equation (1) using the MATLAB function ode 45 with low tolerance. Observe that all the integrators are second-order accurate, and PRK, MCG and LIEMID[EA] show a better accuracy than those of other methods. The solid black line has a $\mathcal{O}\left(h^{2}\right)$ slope.

\section{CONCLUSION}

In this paper, we propose an easy-to-implement numerical experiment that has proven effective in detecting the possible energy drift of a conservative rigid body integrator. The test consists in observing the evolution of the dynamics of a rigid body in an ad hoc static configuration-dependent potential field, obtained perturbing a stable equilibrium set through the introduction of a potential that defines a strong attractive point. We tested various rigid body methods taken from the literature. All these schemes show an energy drift, thus disproving the conjecture on their symplecticity or conjugate symplecticity. This test remarks the importance in long-time integration of symplecticity for a numerical method, as the simulations of the VLV method confirm. Further theoretical investigations are required to understand why this test is effective in highlighting a drift and to explore the possibility of extending the test on a generic Lie group other than $\mathrm{SO}(3)$.

\section{ACKNOWLEDGEMENTS}

We want to heartily express our gratitude to Nawaf Bou-Rabee for his precious suggestions and contributions to this work. We wish to thank Petr Krysl and Melvin Leok for the useful discussions. This paper is dedicated to the memory of Professor Jerry Marsden, to which we are in debt for his encouragement, excellent teaching, and contributions in the field. AS acknowledges the support of CONAV/FCT-PT (PTDC/EEACRO/113820/2009), Co3-AUVs (EU FP7 no.231378), and FCT-ISR/IST plurianual funding program.

\section{REFERENCES}

1. Lewis D, Simo JC. Conserving algorithms for the dynamics of Hamiltonian systems on Lie groups. Journal of Numerical Science 1994; 4:253-299.

2. Moser J, Veselov AP. Discrete versions of some classical integrable systems and factorization of matrix polynomials. Communications in Mathematical Physics 1991; 139:217-243.

3. Reich S. Momentum conserving symplectic integrators. Physica D: Nonlinear Phenomena 1994; 76:375-383.

4. McLachlan RI, Scovel C. Equivariant constrained symplectic integration. Journal of Nonlinear Science 1995; 5:233-256.

5. Bou-Rabee N, Marsden JE. Hamilton-Pontryagin integrators on Lie groups. Foundations of Computational Mathematics 2008; 9:197-219. 
6. Saccon A. Midpoint rule for variational integrators on Lie groups. International Journal for Numerical Methods in Engineering 2009; 78:1345-1364.

7. Marsden JE, Ratiu TS. Introduction to Mechanics and Symmetry, Volume 17 of Texts in Applied Mathematics. Springer: London, 1999.

8. Benettin G, Giorgilli A. On the Hamiltonian interpolation of near to the identity symplectic mappings with applications to symplectic integration algorithms. Journal of Statistical Physics 1994; 74:1117-1143.

9. Simo JC, Vu-Quoc L. On the dynamics in space of rods undergoing large motions - a geometrically exact approach. Computer Methods in Applied Mechanics and Engineering 1988; 66:125-161.

10. Simo JC, Wong TS. Unconditionally stable algorithms for rigid body dynamics that exactly preserve energy and momentum. International Journal for Numerical Methods in Engineering 1991; 31:19-52.

11. Austin MA, Krishnaprasad PS, Wang L. Almost Poisson integration of rigid body systems. Journal of Computational Physics 1993; 107:105-117.

12. Krysl P, Endres L. Explicit Newmark/Verlet algorithm for time integration of the rotational dynamics of rigid bodies. International Journal for Numerical Methods in Engineering 2005; 62:2154-2177.

13. Krysl P. Explicit momentum-conserving integrator for dynamics of rigid bodies approximating the midpoint Lie algorithm. International Journal for Numerical Methods in Engineering 2005; 63:2171-2193.

14. Nukala PKVV, Shelton Jr W. Semi-implicit reversible algorithms for rigid body rotational dynamics. International Journal for Numerical Methods in Engineering 2007; 69:2636-2662.

15. Koziara T, Bićanić N. Simple and efficient integration of rigid rotations suitable for constraint solvers. International Journal for Numerical Methods in Engineering 2010; 81:1073-1092.

16. Hairer E, Lubich C, Wanner G. Geometric Numerical Integration, Springer Series in Computational Mathematics, Vol. 31. Springer: London, 2006.

17. Kane C, Marsden JE, Ortiz M, West M. Variational integrators and the Newmark algorithm for conservative and dissipative mechanical systems. International Journal for Numerical Methods in Engineering 2000; 49:1295-1325.

18. Faou E, Hairer E, Pham T. Energy conservation with non-sympletic methods: examples and counter-examples. BIT Numerical Mathematics 2004; 44:699-709.

19. Bou-Rabee N, Ortolan G, Saccon A. A counterexample showing that the semi-explicit Lie-Newmark algorithm is not variational. Proceedings of the 10th International Conference on Computational and Mathematical Methods in Science and Engineering, CMMSE, 2010; 251-259.

20. Krysl P. Dynamically equivalent implicit algorithms for the integration of rigid body rotations. Communications in Numerical Methods in Engineering 2008; 24:141-156.

21. McLachlan RI, Zanna A. The discrete Moser-Veselov algorithm for the free rigid body, revisited. Foundations of Computational Mathematics 2005; 1:87-123.

22. Veselov AP. Integrable discrete-time systems and difference operators. Translated from Funktsional'nyi Analiz, i Ego Prilozheniya 1998; 22:1-13.

23. Marsden JE, Pekarsky S, Shkoller S. Discrete Euler-Poincaré and Lie-Poisson equations. Nonlinearity 1999; 12:1647-1662.

24. Marsden JE, West M. Discrete mechanics and variational integrators. Acta Numerica 2001; 10:357-514.

25. Taeyoung L, Leok M, McClamroch NH. Lie group variational integrators for the full body problem in orbital mechanics. Celestial Mechanics and Dynamical Astronomy 2007; 98(2):121-144.

26. McLachlan RI, Quispel GRW. Splitting methods. Acta Numerica 2002; 11:341-434.

27. Celledoni E, Fassó F, Säfström N, Zanna A. The exact computation of the free rigid body motion and its use in splitting methods. SIAM Journal on Scientific Computing 2008; 30(4):2084-2112.

28. Ge Z, Marsden JE. Lie-Poisson Hamilton-Jacobi theory and Lie-Poisson integrators. Physics Letters A 1988; 133(3):134-139.

29. Jurdjevic V. Geometric Control Theory. Cambridge University Press: Cambridge, UK, 1997.

30. Reich S. Backward error analysis for numerical integrators. SIAM Journal on Numerical Analysis 1999; 36: 1549-1570.

31. Benettin G, Cherubini AM, Fassò F. A changing-chart symplectic algorithm for rigid bodies and other Hamiltonian systems on manifolds. SIAM Journal of Scientific Computation 2001; 23(4):1189-1203.

32. Hughes TJR. The Finite Element Method. Prentice-Hall: Upper Saddle River, New Jersey, 1987.

33. Bou-Rabee N. Hamilton-Pontryagin integrators on Lie groups. Ph.D. Thesis, California Institute of Technology, 2007.

34. Munthe-Kaas HZ. Runge-Kutta methods on Lie groups. BIT Numerical Mathematics 1998; 38:92-111.

35. Munthe-Kaas HZ. High order Runge-Kutta methods on manifolds. Applied Numerical Mathematics 1999; 29 : $115-127$.

36. Jay L. Symplectic partitioned Runge-Kutta method for constrained Hamiltonian systems. SIAM Journal of Numerical Analysis 1996; 33:368-387.

37. Eng $\varnothing$ K. Partitioned Runge-Kutta methods in Lie-group setting. BIT Numerical Mathematics 2003; 43:21-39.

38. Crouch PE, Grossman R. Numerical integration of ordinary differential equations on manifolds. Journal of Nonlinear Science $1993 ; \mathbf{3}: 1-33$.

39. Owren B, Marthinsen A. Runge-Kutta methods adapted to manifolds and based on rigid frames. BIT Numerical Mathematics 1999; 39:116-142.

40. Marsden JE, Scheurle J. The reduced Euler-Lagrange equations. Fields Institute Communications 1993; 1:139-164. 